\title{
CARACTERÍSTICAS FISICOQUÍMICAS, DE ÁCIDOS GRASOS Y AMINOÁCIDOS EN CORDEROS DE OVELLA GALEGA A 45 DÍAS
}

\author{
PHYSICO-CHEMICAL PROPERTIES, FATTY ACIDS AND AMINO ACIDS FOUND IN 45 \\ DAYS OLD OVELLA GALEGALAMBS
}

\begin{abstract}
Adán, S. ${ }^{1 \star}$, Fernández, M. ${ }^{2}$, Domínguez, B. ${ }^{3}$, Rivero, C.J. ${ }^{4}$, Justo, J.R. ${ }^{1}$, Arias, A. ${ }^{1}$, GarcíaFontán, M.C. ${ }^{2}$, Lorenzo, J.M. ${ }^{2}$, Lama, J.J. ${ }^{1}$, López, C. ${ }^{4}$, Rois, D. ${ }^{1}$, Feijóo, J. ${ }^{4}$ y Franco, D. ${ }^{2}$

${ }^{1}$ Federación de Razas Autóctonas de Galicia. Fontefiz. Coles. Ourense. España. *asovega@asovega.es ${ }^{2}$ Fundación Centro Tecnológico de la Carne. San Cibrao das Viñas. Ourense. España.

${ }^{3}$ Instituto Ourensán de Desenvolvemento Económico. Ourense. España.

${ }^{4}$ Centro de Recursos Zooxenéticos de Galicia. Fontefiz. Coles. Ourense. España.
\end{abstract}

\section{PALABRAS CLAVE ADICIONALES}

Lechal. Raza ovina en peligro de extinción.

\section{RESUMEN}

Se han analizado los parámetros fisicoquímicos, y el perfil de aminoácidos y de ácidos grasos en corderos de Ovella Galega alimentados exclusivamente con leche materna y sacrificados a los 45 días. Debe destacarse el bajo porcentaje de grasa intramuscular $(0,62 \%)$ y dureza media-baja $\left(4 \mathrm{~kg} / \mathrm{cm}^{2}\right)$. El perfil aminoacídico se aproxima al de una proteína de alta calidad. Entre los ácidos grasos se aprecia alto porcentaje de insaturados (55\%) tanto mono como poliinsaturados, y menor de saturados $(44,82 \%)$. La relación $\omega_{6} / \omega_{3,}(1,88)$ es adecuada.

\section{SUMMARY}

There were analyzed the physico-chemical properties and the profile of amino acids and fatty acids found in lambs of the Ovella Galega breed fed solely on their mother's milk and slaughtered at 45 days. The results show a low percentage of intramuscular fat $(0.62 \%)$ and lower-middle hardness $\left(4 \mathrm{~kg} / \mathrm{cm}^{2}\right)$. The amino acids' profile is similar to that of a high quality protein. Concerning the fatty acids' profile, the study shows a high percentage of unsaturated (55\%), both mono and polyunsaturated, versus a lower percentage of the saturated ones (44.82\%), apart from a well proportioned ratio $\omega_{6} / \omega_{3},(1,88)$.

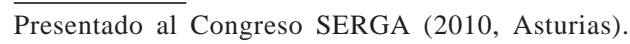

\section{AdDITIONAL KEYWORDS}

Suckling lambs. Endangered sheep breed.

\section{INTRODUCCIÓN}

La raza Ovella Galega, autóctona de Galicia se encuentra catalogada como en peligro de extinción por el Real Decreto 2129/2008, de 26 de diciembre, por el que se establece el Programa nacional de conservación, mejora y fomento de las razas ganaderas, por lo cual está sometida a un Programa de Conservación y Recuperación (Fernández et al., 2001; Rivero et al., 2001). Esta raza de formato pequeño, es criada en condiciones de régimen extensivosemiextensivo, y está orientada hacia la producción de carne. En este estudio se pretende caracterizar la carne de cordero de Ovella Galega, mediante análisis de sus características fisicoquímicas así como de los perfiles de ácidos grasos y aminoácidos.

\section{MATERIAL Y MÉTODOS}

Se han seleccionado 10 corderos machos de parto simple inscritos en el Libro Genealógico de la raza (Xunta de Galicia, 2000), permaneciendo con la madre y alimentados sólo con leche materna hasta la edad de sacrificio de 45 días. Tras el sacrificio y posterior despiece, en el músculo 
Longissimus dorsi, se ha determinado el $\mathrm{pH}$ (24h), la capacidad de retención de agua (CRA) (Honikel, 1987), composición química (proteína, grasa, cenizas y humedad según normas ISO: R-1442:1997, R-1443:1973, R-937:1978, R-936:1998 respectivamente), hidroxiprolina (Presidencia del Gobierno, 1979), minerales (Lorenzo et al. 2003) y textura instrumental (AMSA, 1995). Se realizó una extracción y determinación de los aminoácidos libres mediante cromatografía líquida de alta eficacia (Alonso et al., 1994). Para el perfil de ácidos grasos, se ha extraído la grasa intramuscular según método Folch et al. (1957), la formación de ésteres metílicos se ha realizado por el método de transesterificación (Shehata et al., 1970), posteriormente el análisis de identificación y cuantificación de los ácidos grasos se ha realizado mediante cromatografía de gases

Tabla I. Características fisicoquímicas y minerales en corderos de Ovella Galega con 45 días). (Physico-chemical and mineral properties found in Ovella Galega lambs at an age of 45 days).

\begin{tabular}{lcccr}
\hline & Mínimo & Máximo & Media & DE \\
\hline pH (24 h) & 5,67 & 5,84 & 5,77 & 0,05 \\
Humedad (\%) & 75,19 & 77,56 & 76,50 & 0,75 \\
Proteína (\%) & 19,87 & 20,90 & 20,31 & 0,34 \\
Grasa (\%) & 0,28 & 0,95 & 0,62 & 0,22 \\
Cenizas (\%) & 1,25 & 1,60 & 1,37 & 0,11 \\
CRA & 15,30 & 34,63 & 23,94 & 5,86 \\
Colágeno (\%) & 0,36 & 0,66 & 0,49 & 0,09 \\
Dureza (kg/cm²) & 2,34 & 5,67 & 4,00 & 1,10 \\
Ca (mg/100g) & 1,97 & 3,22 & 2,50 & 0,46 \\
Cu (mg/100g) & 0,27 & 0,45 & 0,33 & 0,05 \\
Fe (mg/100g) & 2,02 & 2,58 & 2,26 & 0,21 \\
$\mathrm{~K}(\mathrm{mg} / 100 \mathrm{~g})$ & 131,00 & 193,00 & 174,70 & 17,63 \\
$\mathrm{Mg}(\mathrm{mg} / 100 \mathrm{~g})$ & 13,70 & 18,20 & 16,16 & 1,46 \\
$\mathrm{Mn}(\mathrm{mg} / 100 \mathrm{~g})$ & 0,02 & 0,04 & 0,03 & 0,01 \\
$\mathrm{Na}(\mathrm{mg} / 100 \mathrm{~g})$ & 71,00 & 113,40 & 90,69 & 14,76 \\
$\mathrm{P}(\mathrm{mg} / 100 \mathrm{~g})$ & 119,00 & 145,00 & 135,40 & 8,81 \\
$\mathrm{Zn}(\mathrm{mg} / 100 \mathrm{~g})$ & 1,89 & 2,38 & 2,15 & 0,16 \\
& & & &
\end{tabular}

DE: desviación estándar; CRA: \% pérdidas por cocción; $\mathrm{n}=10$.
Tabla II. Perfil aminoacídico (mg/100 g) en corderos de Ovella Galega con 45 días $(n=10)$. (Amino acids' profile found in Ovella Galega lambs, 45 days of age $(n=10))$.

\begin{tabular}{lcccc}
\hline & Mínimo & Máximo & Media & DE \\
\hline Hidroxiprolina & 5,06 & 8,24 & 6,96 & 1,11 \\
Ácido aspartico & 0,69 & 1,35 & 1,03 & 0,19 \\
Serina & 2,02 & 4,89 & 3,47 & 0,82 \\
Ácido glutámico & 2,94 & 5,82 & 4,65 & 0,88 \\
Glicina & 8,48 & 49,48 & 17,85 & 12,41 \\
Histidina & 14,42 & 27,73 & 19,87 & 4,25 \\
Taurina & 20,91 & 113,71 & 83,90 & 25,53 \\
Arginina & 129,51 & 207,63 & 161,47 & 28,34 \\
Treonina & 84,07 & 225,60 & 111,46 & 41,72 \\
Alanina & 17,03 & 40,86 & 24,36 & 7,13 \\
Prolina & 5,35 & 11,28 & 9,09 & 1,78 \\
Cistina & 1,29 & 3,25 & 2,11 & 0,60 \\
Tirosina & 2,27 & 4,47 & 2,98 & 0,78 \\
Valina & 1,83 & 4,44 & 3,19 & 0,65 \\
Metionina & 0,68 & 1,52 & 1,03 & 0,26 \\
Lisina & 1,87 & 3,34 & 2,68 & 0,41 \\
Isoleucina & 1,60 & 2,63 & 2,04 & 0,30 \\
Leucina & 2,30 & 4,85 & 3,46 & 0,72 \\
Fenilalanina & 1,55 & 2,89 & 2,16 & 0,40 \\
& & & & \\
\hline
\end{tabular}

con detector FID (condiciones cromatográficas descritas por Kramer and Zhou, 2001). Por último se han determinado los índices de ácidos grasos: insaturados /saturados, poliinsaturados/saturados y $\omega_{6} / \omega_{3}$ El análisis de datos se ha realizado con el paquete estadístico SPSS 17.0 para Windows.

\section{RESULTADOSYDISCUSIÓN}

Los resultados se muestran en las tablas I, II y III. Como se puede observar en la tabla I, destaca el bajo porcentaje en grasa que presentan los corderos lechales de Ovella Galega (0,62\%), frente a valores superiores para corderos de aptitud cárnica de otras razas españolas como el 1,87\% para la raza Lacha (Horcada et al., 1994) o extranjeras como, el 2,23\% para la raza italiana Apenninica (Russo et al., 2002). El resto de parámetros concuerdan con los valores

Archivos de zootecnia vol. 60, núm. 231, p. 434. 
Tabla III. Composición en ácidos grasos (\% sobre el total de ésteres metílicos) en corderos de Ovella Galega con 45 días $(n=10)$. (Fatty acids' composition in Ovella Galega lambs at an age of 45 days $(n=10))$.

\begin{tabular}{|c|c|c|c|c|}
\hline & Mínimo & Máximo & Media & $\mathrm{DE}$ \\
\hline C10:0 & 0,00 & 0,27 & 0,17 & 0,10 \\
\hline $\mathrm{C} 12: 0$ & 0,27 & 0,91 & 0,71 & 0,20 \\
\hline C14:0 & 3,47 & 8,28 & 5,88 & 1,38 \\
\hline C15:0 & 0,46 & 0,68 & 0,56 & 0,08 \\
\hline C16:0 & 19,38 & 24,92 & 23,32 & 1,76 \\
\hline C17:0 & 0,72 & 1,00 & 0,83 & 0,10 \\
\hline C18:0 & 11,71 & 15,52 & 13,16 & 1,25 \\
\hline C23:0 & 0,00 & 0,35 & 0,21 & 0,16 \\
\hline C14:1 & 0,00 & 0,25 & 0,18 & 0,08 \\
\hline C15:1 & 0,00 & 0,11 & 0,04 & 0,05 \\
\hline C16:1 & 0,94 & 2,37 & 1,60 & 0,48 \\
\hline C17:1 & 0,00 & 0,86 & 0,41 & 0,31 \\
\hline C18:1n9t & 0,15 & 0,32 & 0,26 & 0,05 \\
\hline TVA C18:1 & 1,28 & 2,57 & 2,01 & 0,47 \\
\hline C18:1n9c & 34,22 & 40,26 & 36,85 & 2,09 \\
\hline C18:2n6c & 1,62 & 10,02 & 6,13 & 2,33 \\
\hline C18:3n3 & 1,08 & 2,81 & 1,90 & 0,50 \\
\hline C20:3n6 & 0,00 & 0,31 & 0,17 & 0,08 \\
\hline $\mathrm{C} 20: 4 \mathrm{n} 6$ & 0,41 & 4,77 & 2,81 & 1,48 \\
\hline C20:5n3 & 0,26 & 2,69 & 1,67 & 0,68 \\
\hline C22:6n3 & 0,16 & 1,71 & 0,97 & 0,45 \\
\hline AGS & 38,67 & 51,35 & 44,82 & 3,59 \\
\hline AGMI & 38,60 & 44,25 & 41,36 & 2,08 \\
\hline AGPI & 3,54 & 21,32 & 13,65 & 5,22 \\
\hline AGI & 47,69 & 60,72 & 55,01 & 3,69 \\
\hline$\omega_{3}$ & 1,50 & 7,04 & 4,70 & 1,61 \\
\hline$\omega_{6}^{3}$ & 2,03 & 14,96 & 9,11 & 3,75 \\
\hline$\omega_{6} / \omega_{3}$ & 1,35 & 2,34 & 1,88 & 0,33 \\
\hline AGPI/AGS & 0,07 & 0,55 & 0,31 & 0,14 \\
\hline AGI/AGS & 0,93 & 1,57 & 1,24 & 0,18 \\
\hline
\end{tabular}

AGS: ácidos grasos saturados; AGMI: ácidos grasos monoinsaturados; AGPI: ácidos grasos

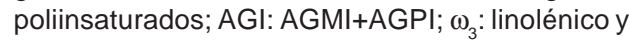
derivados; $\omega_{6}$ : linoleico y derivados.

\section{BIBLIOGRAFÍA}

Almela, E., Jordán, M.J., Martínez, C., Sotomayor, J.A., Bedia, M. y Bañón, S. 2009. El flavor de la obtenidos por otros autores (Cañeque et al., 2005). En la tabla II se muestra el perfil aminoacídico, donde se observa que las cantidades de aminoácidos esenciales se aproximan a los valores de una proteína de alta calidad (Martín, 1997). Como se puede ver en la tabla III, se ha obtenido un alto porcentaje de ácidos grasos insaturados, así como un alto porcentaje de aminoácidos $\omega_{3}$, y esto es debido en parte al régimen extensivo de explotación en el que se encuentran las madres, alimentadas con pastos, lo que influye en la composición de la leche y que repercute directamente en la composición de ácidos grasos de la carne de corderos lechales (Scerra et al., 2007; Almela et al., 2009). La relación $\omega_{6} / \omega_{3}$ es adecuada, manteniéndose en valores inferiores a 4/1, lo recomendado en dietas cardiosaludables (Simopoulos, 2002).

\section{CONCLUSIONES}

Los resultados concuerdan con el de corderos lechales criados en condiciones sostenibles de explotación obteniéndose carnes poco grasas, poco duras, con proteínas de alta calidad y un perfil en ácidos grasos donde destaca su alto porcentaje en ácidos grasos insaturados.

Este trabajo, que forma parte de otro más amplio, nos permite obtener información para ampliar la caracterización y diferenciación de la carne procedente de corderos de la raza Ovella Galega.

\section{AGRADECIMIENTOS}

Este trabajo forma parte del proyecto FEADER 2008/19, cofinanciado por FEADER, Consellería do Medio Rural de la Xunta de Galicia y Ministerio de Medio Ambiente y Medio Rural y Marino del Gobierno de España.

carne cocinada de cordero. Eurocarne, 178: 112. 


\section{ADÁN ETAL.}

Alonso, M.L., Álvarez, A.I. and Zapico, J. 1994. Rapid analysis of free amino acids in infant foods. J. Liquid Chromat., 17: 4019-4030.

AMSA. 1995. Research guidelines for cookery, sensory evaluation and instrumental tenderness measurements of fresh meat. American Meat Science Association. Savoy, IL.

Cañeque, V. y Sañudo, C. 2005. Estandarización de las metodologías para evaluar la calidad del producto (animal vivo, canal, carne y grasa) en los rumiantes. Monografías INIA. Serie Ganadera, $\mathrm{n}^{\circ} 3$.

Fernández, M., Rivero, G., Alonso, M., Rivero, C.J., Pose, H., Justo, J.R., Adán, S., Díaz, R., Rois, D. y Carril, J.A. 2001. Razas autóctonas de Galicia en peligro de extinción. Servicio de Estudios y Publicaciones de la Consellería de Política Agroalimentaria e Desenvolvemento Rural. Xunta de Galicia.

Folch, J., Lees, M. and Stanley, G.H.S. 1957. A simple method for the insolation and purification of total lipides from animal tissues. J. Biol. Chem., 226: 497-509.

Honikel, K.O. 1987. The water binding of meat. Fleischwirtschaft, 67: 1098-1102.

Horcada, A., Berlain, M.J., Purroy, A., Chasco, J., Lizaso, G., Mendizábal, J.A., Mendizábal, F.J. 1994. Efecto del sexo sobre la calidad de la carne de los corderos de las razas Lacha y Aragonesa. ETSIA. Universidad Pública de Navarra. Pamplona.

ISO. International Organization for Standarization. 1973. Determination of total fat content. ISO 1443:1973 Standard. In: International Standards Meat and Meat Products. Ginebra. Suiza.

ISO. International Organization for Standarization. 1978. Determination of nitrogen content. ISO 937: 1978 Standard. In: International Standards Meat and Meat Products. Ginebra. Suiza.

ISO. International Organization for Standarization. 1997. Determination of moisture content. ISO 1442:1997 Standard. In: International Standards Meat and Meat Products. Ginebra. Suiza.

ISO. International Organization for Standarization. 1998. Determination of ash content. ISO
936:1998 Standard. In: International Standards Meat and Meat Products. Ginebra. Suiza.

Kramer, J.K.G. and Zhou, J. 2001. Conjugated linoleic acid and octadecenoic acid: Extraction and isolation of lipids. Europ. J. Lipid Sci. Technol., 103: 594-599.

Lorenzo, J.M., Prieto, B., Carballo, J. and Franco, I. 2003. Compositional and degradative changes during the manufacture of dry-cured 'lacón'. J. Sci. Food Agric., 83: 593-601.

Martín, G. 1997. Tabla de composición de alimentos. Nutricia S.A. Madrid.

Presidencia de Gobierno. 1979. Orden de 31 de julio de 1979. Métodos oficiales de análisis de aceites y grasas, productos cárnicos, cereales y derivados, fertilizantes, productos fitosanitarios, productos lácteos, piensos, aguas y productos derivados de la uva. Boletín Oficial del Estado no 207, de 29 de agosto de 1979.

Rivero, C.J., Rivero, G., García, J., Pose, H., Justo, J.R. y Fernández, M. 2001. Actuaciones para la conservación de la Ovella Galega. Arch. Zootec., 50: 259-264.

Russo, C., Preziuso, G. and Verità, P. 2002. EU carcass classification system: carcass and meat quality in light lambs. Department of Animal Production. University of Pisa.

Scerra, M., Caparra, P., Foti, F., Galofaro, V., Sinatra, M.C. and Scerra, V. 2007. Influence of ewe feeding systems on fatty acid composition of suckling lambs. Meat Sci., 76: 390-394.

Shehata, A.J., De Man, J.M. and Alexander, J.C. 1970. A simple and rapid method for the preparation of methyl esters of fats in milligram amounts for gas chromatography. Can. Inst. Food Sci. Technol. J., 3: 85-89.

Simopoulos, A.P. and Artemis, P. 2002. The importance of the ratio of omega-6/omega-3 essential fatty acids. Biomed. Pharmacother. 56: 365-379.

Xunta de Galicia. 2000. Orden de 27 de septiembre de 2000 , por la que aprueba la reglamentación específica del Libro Genealógico de la raza ovina gallega. Diario Oficial de Galicia $n^{\circ} 206$, de 24 de octubre: 14.395-14.398.

Archivos de zootecnia vol. 60, núm. 231, p. 436. 